\title{
NOTE
}

\section{Algebra and Logics}

\author{
Tika Ram Bhusal \\ Department of Mathematics, Patan Multiple campus, \\ Tribhuvan University, Kathmandu, Nepal \\ Email:tikrambhusal@gmail.com
}

\begin{abstract}
The context of abstractness in Algebra depends on the study of logics. Logics when dealt in some meani ngful way may give challenges to the game of theories and theorems. We have tried to find some examples which should be with the spirit of trends of thoughts. Axioms are basic assumptions but they should be consistent throughout the problem. In the study of logics, statements should satisfy certain property. We cannot introduce any statement in the curriculum dictatorially.
\end{abstract}

Keywords: Algebra, logics, axioms, statements, lemmas, Fermat's last theorem, Falting's theorem

\section{INTRODUCTION}

Few examples of axioms, theorems, statements and lemma are stated in this report. Starting with the definitions followed by consistency is discussed taking with different examples.

\section{Definitions}

$D_{1}$ : Axioms- In general, axioms are basic assumptions which are not proved, it is only necessary that they be consistent throughout the problem.

$D_{2}$ : Theorems- The conclusion obtained from the axioms by logical reasoning are called Theorem.

$D_{3}$ : Statements- Sentences which have the property of being either true or false are called statements.

$D_{4}$ : Lemmas - A lemma is a statement introduced to help in the proof of a theorem.

$D_{5}$ : Invalid statements - If one example fails out the intrinsic property of statement of theorem or problem, we call such statement as invalid statement. Invalid statement cannot satisfy the definition of statement.

Here we are going to discuss on two topics. The fir st one is basic assumptions (Axioms) and the other one is statements.

\section{Basic assumptions}

Let $f: A \rightarrow B$ be a function. If $\mathrm{A}$ is partitioned by disjoint sets $P_{i}$, each set $P_{i}$ consists of $\mathrm{n}$ elements and each of the n elements of $P_{i}$ is mapped to single element of $\mathrm{B}$. Then we say that $\mathrm{f}$ is $\mathrm{n}$ to 1 function. This is a definition and counts as basic assumption (Axiom) for further studies. It means A contains $\mathrm{m} n$ elements; $\mathrm{m}$, $\mathrm{n} \in \mathrm{N}, \mathrm{N}$ is the set of positive integers.

This axiom is not consistent throughout the discourse of mathematics.

\section{Examples}

(a) Let $Z$ be the set of integers:

(i) Let $f: Z \rightarrow Z$ be a function such that $f(x)=0, \mathrm{x} \in x \in Z$, this is $\infty$ to 1 function.

(ii) Let $f: Z \quad Z$ be a function such that $f(x)=r, \mathrm{x}=2 \mathrm{n}+\mathrm{r}, 0 \leq \mathrm{r}<2, \mathrm{n}, \mathrm{r} \in \mathrm{Z}$, this is $\infty$ to 1 function.

(iii) Let $f: Z \rightarrow Z$ be a function such that $f(x)=r, \mathrm{x}=5 \mathrm{n}+\mathrm{r}, 0 \leq \mathrm{r}<5, \mathrm{n}, \mathrm{r} \in \mathrm{Z}$, this is $\infty$ to 1 function.

(b) The above mentioned axioms (definitions) are not consistent throughout above three examples (i), (ii) and (iii).

(c) Let $\mathrm{R}$ be the set of real numbers, taking the sine function sin: $R \rightarrow R, \mathrm{n}$ to 1 is possible, but the case becomes ambiguous because $\mathrm{R}$ is uncountable and its every close interval is uncountable. It may 
challenge the definition of function. This example is also not consistent with the axiom (definition).

\section{Statements}

When dealing with an implication $p \square q$ as a statement of theorem or problem, it is customary to refer to $p$ as the hypothesis (assumption) and to $\mathrm{q}$ as the conclusion (or a statement to be proved). Main focus is on the statement of popularly known Fermat's last theorem, which is a conjecture. The validity of its statement is discussed.

\section{Motivations}

Motivation (a): It is well known statement that "square of an even integer is an even integer", this is a valid statement. Study of logics has given us another statement of theorem (lemma).

"If $a^{2}$ is even integer then a is an even integer".

Let's state it more properly by giving the name $S_{1}$

$S_{1}$ : In the universal set $\mathrm{Z}$ " if $a^{2}$ is even then a is even".

Let's give counter example for it.

If $a^{2}$ is even then we can suppose $a^{2}=2 m$,

$m \in \mathrm{Z}$ Now, if $\mathrm{m}=1$ then $a^{2}=2.1=2$.

There doesn't exist any $a \in \mathrm{Z}$ such that $a^{2}=2$.

Similarly, if $\mathrm{m}=-1$ then $a^{2}=2$. $(\square 1)=\square 2=2$.

There doesn't exist any $a \in Z$ such that $a^{2}=-2$

We conclude that, if $a^{2}$ is even, then, $1 \notin Z$. It fails

to say that "if $a^{2}$ is even integer then a is an even integer".

Further, in the universal set $\mathrm{Z}$, we can split it into two sets $\mathrm{E}$ and $\mathrm{O}$ such that $\mathrm{E} \cup \mathrm{O}=\mathrm{Z}$ and $\mathrm{E} \cap \mathrm{O}$ $=\varphi, E$ is set of even integers and $O$ is set of odd

integers. $a^{2}$ cannot cover all elements of $\mathrm{E}$, so no question arises to see the Truth Table. This counter example suggests that the statement $S_{1}$ is invalid statement of theorem or lemma or problem.

Motivation (b): Let $n \in$ such that $\mathrm{n}>1$. We define $U(\mathrm{n})$ to be the set of all positive integers less than $\mathrm{n}$ and relatively prime to $\mathrm{n}$, then $U(\mathrm{n})$ is a group under multiplication modulo $\mathrm{n}$.

$U\left(2^{n}\right), \mathrm{n}$ is positive integer, is also defined in the same way.

Statement of the problem: Prove $U\left(2^{n}\right)$, is not cyclic group for all $n \geq 3$.

Let's prove it using logics.

Proof:

Let " $U\left(2^{n}\right)$ is cyclic for all $n \geq 3$ " be the statement $\mathrm{S}$ and $\mathrm{S}$ be valid for all $n \geq 3$.
Now, when $\mathrm{n}=3, U\left(2^{3}\right)=U(8)=\{1,3,5,7\}$. We see that,

$$
\begin{aligned}
& \left\{3,3^{2}, 3^{3}, 3^{4}\right\}=\{1,3\} \neq U\left(2^{3}\right) \\
& \left\{1,1^{2}, 1^{3}, 1^{4}\right\}=\{1\} \neq U\left(2^{3}\right) \\
& \left\{5,5^{2}, 5^{3}, 5^{4}\right\}=\{1,5\} \neq U\left(2^{3}\right) \\
& \left\{7,7^{2}, 7^{3}, 7^{4}\right\}=\{1,7\} \neq U\left(2^{3}\right)
\end{aligned}
$$

This shows that $U\left(2^{3}\right)$ is not cyclic. Above illustration implies that $\mathrm{S}$ is not true for $n=3$. This implies that $\mathrm{S}$ is invalid statement by definition $D_{5}$. This implies that $U\left(2^{n}\right)$ is not cyclic for all $n \geq 3$.

Hence proved

Motivation(c): Case: It is given that out of three numbers $\mathrm{x}, \mathrm{y}$ and $\mathrm{z}$, if at least one is integer. Let 's take three statements " $\{x, y, z\}$ has integers" be the statement for $S_{2}$ has " $\{x, y, z\}$ has at least one integer" be the statement for $S_{3}$ " $\{\mathrm{x}, \mathrm{y}, \mathrm{z}\}$ has no integers" be the statement $\mathrm{S}_{4}$.

Statements $\mathrm{S}_{2}$ and $\mathrm{S}_{4}$ are invalid for this case.

Reason: In particular, suppose $\mathrm{x}$ is an integer and $\mathrm{y}, \mathrm{z}$ are non integers. It will be meaningless to say “ $\{\mathrm{x}, \mathrm{y}, \mathrm{z}\}$ has integers"

Similarly, since $\mathrm{y}, \mathrm{z}$ are non integers and $\mathrm{x}$ is an integer. It will be meaningless to say that" $\{\mathrm{x}, \mathrm{y}, \mathrm{z}\}$ has no integers". That is why statements $\mathrm{S} 2$ and $\mathrm{S} 4$ are invalid for this case.

\section{Invalidity of statement of Fermat's last theorem or conjecture or problem:}

The statement given by Fermat in translated form is as follows:

"It is impossible to separate a cube into two cubes , a fourth power into two fourth powers or generally any power above the second into two powers of the same degree".

If universal discourse is the set of positive whole numbers and Faltings theorem is not considered, then this statement according to the definition is valid.

In the present context, statement given in times magazine, July 5, 1993 by Michael D. Lemonick is as follows, Fermat's last theorem:

"The equation $x^{n}+y^{n}=z^{n}$, where $\mathrm{n}$ is an integer greater than 2 , there is no solution in positive integers".

In the text of Michael Artin, the statement is as follows:

The equation $x^{n}+y^{n}=z^{n}$, for $n \geq 3$ has no integer solution $\mathrm{x}, \mathrm{y}, \mathrm{z}$, except for the trivial solutions in which one of the variable is zero. 
Let's continue the discussion, suppose equation " $\mathrm{f}(\mathrm{x})=0$ has integer solution" be statement $\mathrm{S}_{5}$

Suppose equation " $\mathrm{f}(\mathrm{x})=0$ has no integer solution" be statement $\mathrm{S}_{6}$. If $\mathrm{S}_{6}$ is true then $\mathrm{S}_{6}$ will be false.

Similarly, if $S_{5}$ is true then $S_{6}$ will be false, because equation $\mathrm{f}(\mathrm{x})=0$ has no integer solution means that each and every solution is not an integer. And in the same way $f(x)=0$ has integer solution means each and every solution is an integer.

Now let's return to Fermat's conjecture (last theorem) Let's suppose that $\mathrm{F}$ be the statement, $" x^{n}+y^{n}=z^{n}$ has integer solution in positive integers for $n>2 "$.

$\mathrm{F}$ is true for $\mathrm{n}>2, \mathrm{n}$ is an integer.

Now this assumption implies that $\mathrm{F}$ can be true for $\mathrm{n}=4$

Let's check for $\mathrm{n}=4$. We have $x^{n}+y^{n}=z^{n}$ and let $x^{2}=p$, $y^{2}=q, z^{2}=r$

Then we will have $p^{2}+q^{2}=r^{2}$.

(A) If $\quad x=2, \quad y=\sqrt{3}, \quad z=\sqrt{5} \quad$ then $x^{4}+y^{4}=z^{4}$ is satisfied, that means we have found set $\{\mathrm{x}, \mathrm{y}, \mathrm{z}\}$ such that $x \in Z, y \notin Z, z \notin Z$ this case violates the statements $S_{2}$ and $S_{4}$.

(B) If $\mathrm{x}=2, \mathrm{y}=5, \mathrm{z}=\mathrm{r}$ where $r^{4}=641$ then $x^{4}+y^{4}=z^{4}$ is satisfied.

This shows that we have found set $\{\mathrm{x}, \mathrm{y}, \mathrm{z}\}$ such that $x \in Z, y \in Z, z \notin Z$ this case violates the statements $S_{2}$ and $S_{4}$.

(C) If $x=\sqrt{5}, y=2 \sqrt{3}, z=\sqrt{13} \quad$ then, $x^{4}+y^{4}=z^{4}$ is satisfied. This shows that we have found set $\{x, y, z\}$ such that $x \notin Z, y \notin z$ and $z \notin Z$, which supports the statement $\mathrm{S}_{4}$.

By definition $\mathrm{D}_{5}$, the statement $" x^{n}+y^{n}=z^{n}$ has positive integer solution for $n>2, \mathrm{n}$ is an integer" is invalid. If above statement is not valid then the statement $" x^{n}+y^{n}=z^{n}$ has no positive integer solution for $n>2, \mathrm{n}$ is an integer" is also not valid.

It is because of $\mathrm{S}_{5}$ and $\mathrm{S}_{6}$.

$S_{5}$ is related with $S_{2}$ and $S_{6}$ is related to $S_{4}$. Hence the statement of Fermat's last theorem is not valid.

Further, Faltings theorem, implies that for each integer $\mathrm{n}>2$ the Fermat equation $x^{n}+y^{n}=z^{n}$ has at most a finite number of solutions. For invalidity, single case of $n$ is sufficient.

Faltings theorem is sufficient and strong support for the above claim of invalidity.

After 350 years, new logics have made old statement more ambiguous statement.

we would like to suggest clear statement so that ambiguity will be removed.

"IF Z, the set of integers, is the universal set of discourse and $\mathrm{x}, \mathrm{y}, \mathrm{z}$ belong to the set $\mathrm{Z}$, then the statement $x^{n}+y^{n}=z^{n}$ is invalid for $n>2$, $\mathrm{n}$ belong to the set Z."

\section{CONCLUSION}

According to this new statement, the theorem need not be proved; Andrew Wiles should accept the weakness. For the studies especially in mathematics, statement play important role. Statement should agree with the whole domain of pure mathematics, interplay of pure and applied mathematics will take less time for the students to understand the things, specially who believe in fundamental concepts.

\section{REFERENCES}

Algebra, Michael Artin, Prentice Hallof India

Abstract Algebra, Joong Fang, Schaums Publishing Company.

Topics in Algebra, IN Herstein,Wiley Eastern Lt.,India

Topology, James R Munkres, PrenticeHall of India

www.google.com 